S. KAWAI

KODAI MATH. J.

9 (1986), $117-122$

\title{
A REMARK ON THE STABILITY OF YANG-MILLS CONNECTIONS
}

\author{
By SHIGEo KAWAI
}

\section{$\S 1$. Introduction.} theorem.

The purpose of this paper is to prove a generalization of the following

Theorem (J. Simons). If $n>4$, every stable Yang-Mills connection on $S^{n}$ is flat.

If we take tangential components of restrictions of parallel vector fields on $\boldsymbol{R}^{n+1}$ to $S^{n}$, they are conformal vector fields on $S^{n}$. These vector fields are used to construct an infinitesimal deformation for which the second variation is negative. It is believed that the crucial property is the plentifullness of conformal vector fields on $S^{n}$. In fact, Parker [6] gave a unified treatment of this theorem, the relating result for harmonic maps ([7]) and the result of Lawson and Simons concerning currents in $S^{n}$ ([3]) from the viewpoint of conformal geometry.

We show in this paper that the role of conformal vector fields is not essential. Our result is the following.

Theorem. Let $M$ be a compact n-dimensional submanifold of $\boldsymbol{R}^{n+1}$. Suppose that the principal curvatures $\lambda_{1}, \cdots, \lambda_{n}$ of $M$ satisfy the inequalities

$$
2 \delta /(n-2)<\lambda_{\jmath} \leqq \delta \quad(j=1,2, \cdots, n)
$$

everywhere for some $\delta>0$. Then any stable Yang-Mills connection on $M$ is flat.

We also use the vector fields on $M$ obtained by taking tangential component of parallel vector fields on $\boldsymbol{R}^{n+1}$. They are not necessarily conformal vector fields on $M$.

It should be noted that a similar result for harmonic maps was proved by Y. L. Pan and a generalization of the result of Lawson and Simons for stable currents in $S^{n}$ was obtained by Mori [4] and recently improved by Ohnita [5].

Received April 1, 1985 


\section{§. Preliminaries.}

In this section, we prepare some lemmas which will be used in the later section. Their proofs are straightforward and are omitted.

Let $M$ be an $n$-dimensional submanifold of the Euclidean space $\boldsymbol{R}^{n+1}$ which is included in no proper affine subspace of $\boldsymbol{R}^{n+1}$, and $\nu$ be a unit normal vector field on $M$. We consider on $M$ a special space of vector fields

$$
\mathcal{V}=\left\{\operatorname{grad}\left(\left.f\right|_{M}\right) \mid f: \boldsymbol{R}^{n+1} \rightarrow \boldsymbol{R}: \text { linear }\right\} .
$$

There is a natural isomorphism $\boldsymbol{R}^{n+1} \rightarrow \overline{\mathcal{V}}$ which associates to each $V \in \boldsymbol{R}^{n+1}$ the vector field $v$ given by

$$
v(x)=V-\langle V, \nu(x)\rangle \nu(x)
$$

for $x \in M$.

LEMMA 1. For every $v \in \mathcal{V}$ and every $X \in T M$, we have

$$
\nabla_{X} v=\langle V, \nu\rangle A X
$$

where $A$ denote the second fundamental form in the direction of $\nu$.

LEMma 2. Let $\left(e_{1}, \cdots, e_{n}\right)$ be an orthonormal frame of $T_{p} M$. Then denoting by $\nabla^{*}$ the adjoint of $\nabla$, we have for every $v \in \mathcal{C}$,

$$
\nabla^{*} \nabla v=\sum_{\imath}\left\langle v, A e_{\imath}\right\rangle A e_{i}-\langle V, \nu\rangle \sum_{\imath}\left(\nabla_{e_{i}} A\right)\left(e_{\imath}\right) .
$$

LEMma 3. Let $\lambda_{1}, \cdots, \lambda_{n}$ be eigenvalues of $A$ at $p \in M$, and $\left(e_{1}, \cdots, e_{n}\right)$ be an orthonormal frame of $T_{p} M$ which consists of eigenvectors for $\lambda_{1}, \cdots, \lambda_{n}$. Then we have

$$
\begin{aligned}
& R\left(e_{\jmath}, e_{k}\right) e_{l}=\lambda_{\jmath} \lambda_{k}\left(\delta_{k l} e_{j}-\delta_{\jmath l} e_{k}\right), \\
& \operatorname{Ric}\left(e_{k}\right)=\left\{\lambda_{k}\left(\lambda_{1}+\cdots+\lambda_{n}\right)-\lambda_{k}^{2}\right\} e_{k}
\end{aligned}
$$

where $R$ and Ric denote the curvature and the Ricci transformation of $M$ respectively.

Note that the isomorphism $\boldsymbol{R}^{n+1} \rightarrow C V$ transfers an inner product to $C V$ which is invariant under the natural action of $O(n+1)$. We shall consider the trace of a certain quadratic form on $\mathscr{V}$.

\section{$\S 3$. Proof of Theorem.}

In this section we follow the notations of Bourguignon and Lawson [1]. Definitions and detailed proofs of formulae can be found in [1].

Let $P$ be a principal $G$-bundle over a compact orientable manifold $M$ where $G$ is a compact Lie group. We fix a $G$-vector bundle $E=P \times{ }_{\rho} \boldsymbol{R}^{N}$, associated to 
$P$ by a faithful orthogonal representation $\rho: G \rightarrow O(N)$, and define the bundle of Lie algebra $\mathfrak{g}_{E}$ by $\mathfrak{g}_{E}=\bigcup_{x \in M} \operatorname{Hom}_{g}\left(E_{x}, E_{x}\right)$. Let $\mathcal{C}_{E}$ denote the space of connections on $E$ induced by connections on $P$. Then $\mathcal{C}_{E}$ is an affine space with $\Omega^{1}\left(\mathfrak{g}_{E}\right)$ as the vector group of transformations. In particular $T_{V}\left(\mathcal{C}_{E}\right) \cong \Omega^{1}\left(\mathfrak{g}_{E}\right)$ for $\nabla \in \mathcal{C}_{E}$.

To each $\nabla \in \mathcal{C}_{E}$ there is associated a curvature 2-form $R^{\nabla}$ in $\Omega^{2}\left(\mathrm{~g}_{E}\right)$ given by the formula

$$
R \nabla(X, Y)=\left[\nabla_{X}, \nabla_{Y}\right]-\nabla_{[X, Y]}
$$

for tangent vectors $X$ and $Y$. We introduce an inner product on the bundle $g_{E}$ as follows. Recall that we have $\mathfrak{g}_{E} \subset \mathfrak{B D}_{E}$, the bundle of skew-symmetric endomorphisms of $E$. Given two endomorphisms $A$ and $B$ of $E_{x}$, we define

$$
\langle A, B\rangle=(1 / 2) \operatorname{trace}\left({ }^{t} A \circ B\right)
$$

The Yang-Mills functional of $\mathscr{M}: \mathcal{C}_{E} \rightarrow \boldsymbol{R}$ is defined to be

$$
\text { y } \mathscr{M}(\nabla)=(1 / 2) \int_{M}\left\|R^{\nabla}\right\|^{2} * 1 \text {. }
$$

Extremals of the Yang-Mills functional are called Yang-Mills connections. A Yang-Mills connection is called stable if second variations are non-negative for all directions (In [1], they are called weakly stable.)

Let us denote by $R$ the curvature of $M$.

Proposition 1 ([1] (3.10) Theorem). For any $\varphi$ in $\Omega^{2}\left(\mathrm{~g}_{E}\right)$, we have

where

$$
\Delta^{\nabla} \varphi=\nabla^{*} \nabla \varphi+\varphi \cdot(\operatorname{Ric} \wedge I+2 R)+\Re \nabla(\varphi)
$$

$$
\begin{gathered}
\Re^{\nabla}(\varphi)(X, Y)=\sum_{\jmath}\left\{\left[R^{\nabla}\left(e_{\jmath}, X\right), \varphi\left(e_{\jmath}, Y\right)\right]-\left[R^{\nabla}\left(e_{\jmath}, Y\right), \varphi\left(e_{\jmath}, X\right)\right]\right\}, \\
\{\varphi \circ(\operatorname{Ric} \wedge I)\}(X, Y)=\varphi(\operatorname{Ric}(X), Y)+\varphi(X, \operatorname{Ric}(Y))
\end{gathered}
$$

and

$$
(\varphi \circ R)(X, Y)=(1 / 2) \sum_{\jmath} \varphi\left(e_{\jmath}, R(X, Y) e_{\jmath}\right)
$$

for $X, Y \in T_{p} M$ with $\left(e_{1}, \cdots, e_{n}\right)$ an orthonormal basis of $T_{p} M$.

Let $\nabla^{t},|t|<\varepsilon$, be a smooth family of connections on $E$ and $V=\Gamma^{0}$ be a Yang-Mills connection.

Proposition 2 ([1] (6.8) Theorem). Let $B=\left.(d / d t) \Gamma^{t}\right|_{t=0} \in \Omega^{1}\left(\mathfrak{g}_{E}\right)$. Suppose that $\delta^{\nabla} B=0$. Then

$$
\left.\left(d^{2} / d t^{2}\right) y \mathscr{M}\left(\nabla^{t}\right)\right|_{t=0}=\int_{M}\langle\mathcal{S} \nabla(B), B\rangle * 1
$$

where

$$
\mathcal{S} \Gamma(B)=\nabla^{*} \nabla B+B \cdot \mathrm{Ric}+2 \Re \nabla(B) .
$$


In the above proposition, $B \circ$ Ric and $\Re^{D}(B)$ are defined as follows :

$$
\begin{gathered}
(B \circ \operatorname{Ric})(X)=B(\operatorname{Ric}(X)), \\
\Re \mathcal{V}(B)(X)=\sum_{\jmath}\left[R^{\nabla}\left(e_{\jmath}, X\right), B\left(e_{j}\right)\right]
\end{gathered}
$$

for $X \in T_{p} M$, where $\left(e_{1}, \cdots, e_{n}\right)$ is an orthonormal frame of $T_{p} M$.

For any $v \in C V$, let us define $B \in \Omega^{1}\left(\mathfrak{g}_{E}\right)$ by $B=i_{v} R^{v}$, i. e., $B(X)=R^{\nabla}(v, X)$ for $X \in T M$. It is easily checked that elements of $C V$ are of gradient type in the sense of [1]. Hence by (7.3) Lemma in [1], $B$ satisfies $\delta^{\nabla} B=0$.

Choose an orthonormal frame $\left(e_{1}, \cdots, e_{n}\right)$ of $T_{p} M$, and let $X \in T_{p} M$. Then we have from the formula (7.9) in [1],

$$
\left(\nabla^{*} \nabla B\right)(X)=\left(\nabla^{*} \nabla R^{\nabla}\right)(v, X)-2 \sum_{j}\left(\nabla_{e_{j}} R^{\nabla}\right)\left(\nabla_{e_{j}} v, X\right)+R^{\nabla}\left(\nabla^{*} \nabla v, X\right) .
$$

Since $\Delta^{\nabla} R^{\nabla}=0$ for Yang-Mills connection $\nabla([1]((2.23))$, we have by Proposition 1 ,

$$
\begin{aligned}
\left(\nabla^{*} \nabla R^{\nabla}\right)(v, X)= & -\left\{R^{\nabla} \cdot(\operatorname{Ric} \wedge I+2 R)\right\}(v, X)-\Re^{\nabla}\left(R^{\nabla}\right)(v, X) \\
= & -R^{\nabla}(\operatorname{Ric}(v), X)-R^{\nabla}(v, \operatorname{Ric}(X))-\sum_{\jmath} R^{\nabla}\left(e_{\jmath}, R(v, X) e_{j}\right) \\
& -\sum_{\jmath}\left\{\left[R^{\nabla}\left(e_{\jmath}, v\right), R^{\nabla}\left(e_{\jmath}, X\right)\right]-\left[R^{\nabla}\left(e_{\jmath}, X\right), R^{\nabla}\left(e_{\jmath}, v\right)\right]\right\} .
\end{aligned}
$$

Hence we get

$$
\begin{aligned}
&\left\langle\mathcal{S}^{\nabla}\left(i_{v} R^{\nabla}\right), i_{v} R^{\nabla}\right\rangle=\sum_{k}\left\langle\left(\mathcal{S}^{\nabla}\left(i_{v} R^{\nabla}\right)\left(e_{k}\right), i_{v} R^{\nabla}\left(e_{k}\right)\right\rangle\right. \\
&=\sum_{k}\left\langle\left\langle\nabla^{*} \nabla\left(i_{v} R^{\nabla}\right)\right)\left(e_{k}\right)+i_{v} R^{\nabla}\left(\operatorname{Ric}\left(e_{k}\right)\right)+2 \Re \nabla\left(i_{v} R^{\nabla}\right)\left(e_{k}\right), i_{v} R^{\nabla}\left(e_{k}\right)\right\rangle \\
&=\sum_{k}\left\langle-R^{\nabla}\left(\operatorname{Ric}(v), e_{k}\right)-R^{\nabla}\left(v, \operatorname{Ric}\left(e_{k}\right)\right)-\sum_{\jmath} R^{\nabla}\left(e_{\jmath}, R\left(v, e_{k}\right) e_{\jmath}\right)\right. \\
& \quad-\sum_{\jmath}\left\{\left[R^{\nabla}\left(e_{\jmath}, v\right), R^{\nabla}\left(e_{\jmath}, e_{k}\right)\right]-\left[R^{\nabla}\left(e_{\jmath}, e_{k}\right), R^{\nabla}\left(e_{\jmath}, v\right)\right]\right\} \\
& \quad-2 \sum_{\jmath}\left(\nabla_{e_{j}} R^{\nabla}\right)\left(\nabla_{e_{j}} v, e_{k}\right)+R^{\nabla}\left(\nabla^{*} \nabla v, e_{k}\right)+R^{\nabla}\left(v, \operatorname{Ric}\left(e_{k}\right)\right) \\
&\left.\quad+2 \sum_{\jmath}\left[R^{\nabla}\left(e_{\jmath}, e_{k}\right), R^{\nabla}\left(v, e_{j}\right)\right], R \nabla\left(v, e_{k}\right)\right\rangle \\
&=\sum_{k}\left\langle-R^{\nabla}\left(\operatorname{Ric}(v), e_{k}\right)-\sum_{\jmath} R^{\nabla}\left(e_{\jmath}, R\left(v, e_{k}\right) e_{j}\right)\right. \\
&\left.-2 \sum_{j}\left(\nabla_{e_{j}} R^{\nabla}\right)\left(\nabla_{e_{j}} v, e_{k}\right)+R^{\nabla}\left(\nabla * \nabla v, e_{k}\right), R^{\nabla}\left(v, e_{k}\right)\right\rangle .
\end{aligned}
$$

We assume that the principal curvatures $\lambda_{1}, \cdots, \lambda_{n}$ of $M$ satisfy the inequalities $\eta \delta \leqq \lambda_{\jmath} \leqq \delta$ everywhere for some $\delta>0,2 /(n-2)<\eta \leqq 1$.

PROPOSITION 3. Considering $Q_{2}(v)=\int_{M}\left\langle\mathcal{S}^{\nabla}\left(i_{v} R^{\nabla}\right), i_{v} R^{\nabla}\right\rangle * 1$ as a quadratıc form 
on $C$, we have

$$
\text { Trace of } Q_{2} \leqq\left(4 \eta \delta^{2}\right)\{2-(n-2) \eta\} \mathscr{M}(\nabla) \text {. }
$$

Proof. Let $e_{1}, \cdots, e_{n}$ be orthonormal vectors in $T_{p} M$ which are eigenvectors of $A$ at $p \in M$ for eigenvalues $\lambda_{1}, \cdots, \lambda_{n}$ respectively. We choose an orthonormal basis $V_{1}, \cdots, V_{n+1}$ of $\boldsymbol{R}^{n+1}$ as follows :

$$
V_{1}=e_{1}, \cdots, V_{n}=e_{n}, \quad V_{n+1}=\nu(p)
$$

where $\nu$ is an unit normal vector field on $M$. Then the associated vector fields $v_{1}, \cdots, v_{n+1}$ satisfy the equalities.

$$
v_{1}(p)=e_{1}, \cdots, v_{n}(p)=e_{n}, \quad v_{n+1}(p)=0 .
$$

Hence the sum of integrands of $Q_{2}\left(v_{1}\right), \cdots, Q_{2}\left(v_{n+1}\right)$ at $p$ is

$$
\begin{aligned}
\sum_{k, l}\langle- & R \nabla\left(\operatorname{Ric}\left(e_{l}\right), e_{k}\right)-\sum_{\jmath} R^{\nabla}\left(e_{\jmath}, R\left(e_{l}, e_{k}\right) e_{j}\right) \\
+ & \left.R \nabla\left(\sum_{l}\left\langle e_{l}, A e_{\imath}\right\rangle A e_{\imath}, e_{k}\right), R \nabla\left(e_{l}, e_{k}\right)\right\rangle \\
=\sum_{k, l}\langle- & \left\{\lambda_{l}\left(\lambda_{1}+\cdots+\lambda_{n}\right)-\lambda_{l}^{2}\right\} R^{\nabla}\left(e_{l}, e_{k}\right) \\
& -\sum_{\jmath} R^{\nabla}\left(e_{\jmath}, \lambda_{l} \lambda_{k} \delta_{k j} e_{l}-\lambda_{l} \lambda_{k} \delta_{l j} e_{k}\right) \\
& \left.+\sum_{l} \lambda_{i}^{2}\left\langle e_{l}, e_{\imath}\right\rangle R^{\nabla}\left(e_{\imath}, e_{k}\right), R^{\nabla}\left(e_{l}, e_{k}\right)\right\rangle \\
=- & \sum_{k, l}\left\{\lambda_{l}\left(\lambda_{1}+\cdots+\lambda_{n}\right)-2 \lambda_{l}^{2}-2 \lambda_{l} \lambda_{k}\right\}\left\|R^{\nabla}\left(e_{l}, e_{k}\right)\right\|^{2} .
\end{aligned}
$$

Since we have for all $k$ and $l$ with $k \neq l$,

and

$$
-\left\{\lambda_{l}\left(\lambda_{1}+\cdots \lambda_{n}\right)-2 \lambda_{l}^{2}-2 \lambda_{l} \lambda_{k}\right\} \leqq \eta \delta^{2}\{2-(n-2) \eta\}
$$

$$
\int_{M} \sum_{k, l}\left\|R^{\nabla}\left(e_{l}, e_{k}\right)\right\|^{2} * 1=2 \int_{M}\left\|R^{\nabla}\right\|^{2} * 1=4 \mathscr{Y M}(\nabla),
$$

the proposition is proved.

Now Theorem can be proved easily by Proposition 3 .

\section{REFERENCES}

[1] J.P. Bourguignon And H.B. Lawson, JR., Stability and isolation phenomena for Yang-Mills fields, Commun. Math. Phys., 79 (1981), 189-230.

[2] J.P. Bourguignon, H.B. Lawson, JR. And J. Simons, Stability and gap phenomena for Yang-Mills fields, Proc. Nat. Acad. Sci. U.S.A., 76 (1979), 1550-1553.

[3] H.B. LAwson, JR. AND J. Simons, On the stable currents and their applications 
to global problems in real and complex geometry, Ann. of Math., 98 (1973), 427-450.

[4] H. MORI, Notes on stable currents, Pacific J. Math., 61 (1975), 235-240.

[5] Y. OHNita, preprint.

[6] T. PARKeR, Conformal fields and stability, Math. Z., 185 (1984), 305-319.

[7] Y.L. XIN, Some results on stable harmonic maps, Duke Math. J., 47 (1980), 609-613.

Department of Mathematics

FACUlTy OF SCIENCE

Kyoto University, Kyoto 606

JAPAN 